\title{
Feature Set Selection for On-Line Signatures Using Selection of Regression Variables
}

\author{
Desislava Boyadzieva and Georgi Gluhchev \\ IICT-BAS, Sofia, Bulgaria \\ d.n.dimitrova@gmail.com, gluhchev@iinf.bas.bg \\ http://iict.bas.bg
}

\begin{abstract}
In this paper we approach feature set selection phase in signature verification by applying the method for selection of regression variables based on Mallows Cp criterion for regression. In this way we identify best feature subsets of various sizes for each user of our database on the basis of his/her ten genuine and ten random forgery on-line signatures. Among these subsets we select the best subset that have $\mathrm{Cp}$ value closest to $p$, where $p$ is the number of regression coefficients. Thus, we obtain for each user the best feature subset of a different size. Our aim is to check whether there are common features among best feature subsets for all users which will justify the removal of the rest features from the initial feature set. The results obtained with the database of 140 signatures collected from fourteen users demonstrated that we cannot restrict to common feature set valid for all users but instead of that we have to consider each user best feature set separately in signature verification.
\end{abstract}

Keywords: Signature verification, feature selection, on-line signatures, selection of regression variables, Mallows $\mathrm{Cp}$ criterion.

\section{Introduction}

Signatures are recognized and accepted modality for authentication. Signature verification is the process of confirming the identity of a user based on the handwritten signature of the user as a form of behavioral biometrics [1]. Different approaches to signature verification are considered in survey paper [3]. Over 100 features used in signature verification are presented in [3].

Since some features demonstrate higher discriminatory capability than others, features selection should be performed after feature extraction. Feature selection is defined as the process of selecting $k$ most discriminatory features out of $p$ available ones $(k \leq p)$ and it aims to identify and remove as much irrelevant and redundant information as possible. A review of the work on feature set selection for signatures is performed in [4].

In this paper we investigate the problem of feature set selection for on-line signatures by applying the method for selection of regression variables based on Mallows Cp criterion to identify best feature subsets of various sizes for each of the fourteen users of our database on the basis of his/her ten genuine and ten 
random forgery signatures. Among these subsets we select the best subset that have $\mathrm{Cp}$ value closest to $\mathrm{p}$, where $\mathrm{p}$ is the number of regression coefficients. Thus, we obtain for each user the best feature subset of a different size. Our aim is to check whether there are common features among best feature subsets for all users which will justify the removal of the rest features from the initial feature set. The results obtained demonstrated that we cannot restrict to common feature set valid for all users but instead of that we have to consider each user best feature set separately in signature verification.

The paper is structured as follows: Section 2 considers the selection of regression variables; Section 3 presents some experimental results and the last section draws some conclusion and gives an outlook for feature work.

\section{Selection of Regression Variables}

Regression analysis is one of the most widely used statistical techniques because it provides simple methods for establishing a functional relationship among variables. It has extensive applications in many subject areas [2]. Variable selection in regression analysis is the problem of deciding the variables to be included in the model such that it provides good forecast.

The formulation of the variable selection problem is as follows. It is assumed [7] that there are $n \geq k+1$ observations on a $k$-vector of input variables $x^{t}=$ $\left(x_{1}, \ldots, x_{k}\right)$, and a scalar response $y$, such that the $j$ th response $j=1, \ldots, n$ is determined by:

$$
y_{j}=\beta_{0}+\sum_{i=1}^{k} \beta_{i} x_{i j}+e_{j} .
$$

The model (1) is frequently expressed in matrix notation as:

$$
Y=X \beta+e .
$$

Here $\mathrm{Y}$ is the $\mathrm{n}$-vector of observed responses, $\mathrm{X}$ is the design matrix of dimension $n \times(k+1), \beta$ is the $(k+1)$-vector of unknown regression coefficients and $e$ is n-vector of the residuals $e_{j}$ assumed to be independent $N\left(0, \sigma^{2}\right)$. Regression parameters or coefficients $\beta_{i}, i=0, \ldots, k$ are unknown constants to be estimated from the data and their estimates are denoted by $b_{i}$. In our experiment we decided to remove the intercept $\beta_{0}$ from the equation. Then, the vector $b$ of Least-Squares estimates of the coefficients vector $\beta$ is given by:

$$
b=\left(X^{T} X\right)^{-1} X^{T} Y .
$$

There is no unique technique for selecting best regression equation. If there are $k$ potential independent variables there are $2^{k}$ possible equations to be considered. Variable selection techniques are the following: best subset, backward elimination, forward selection and stepwise methods [2]. In this paper we consider best subset approach. 
A number of criteria have been proposed for selecting the best subset or subsets of independent variables in linear regression analysis. Mallows proposed $C_{p}$ as a criterion to decide on suitable subset among contending subsets. It is a measure of the standardized total squared error defined as:

$$
C_{p}=\frac{R S S_{p}}{\hat{\sigma}^{2}}-(n-2 p)
$$

In this equation $R S S_{p}$ denotes residual sum of squares for the particular regression with $p$ variables including the intercept, if any. $\hat{\sigma}^{2}$ is an estimate of residual mean square $\sigma^{2}$ for full regression:

$$
\hat{\sigma}^{2}=\frac{1}{n-k} \sum_{j=1}^{n}\left(y_{j}-\sum_{i=1}^{k} \beta_{i} x_{i j}\right)^{2} .
$$

If a model is adequate, i.e. does not suffer from lack of fit, then $E\left(R S S_{p}\right)=$ $(n-p) \hat{\sigma}^{2}$ and the following is true for that model:

$$
E\left(C_{p}\right) \approx p
$$

This means that we expect $C_{p}$ value to be about $p$. A plot of $C_{p}$ versus $p$ displays the adequate models as points close to the line $C_{p}=p$. Subsets with small values of $C_{p}$ and values of $C_{p}$ close to $\mathrm{p}$ are considered good.

Hocking and Leslie [5] further describe a method which allows thus subset to be identified after consideration of only a small fraction of all $\left(\begin{array}{l}k \\ p\end{array}\right)$ possible subsets of size $p$. LaMotte and Hocking [6] modified this algorithm in a way that moderately large problems can be treated with minimum of computation. The algorithm specifies the subset of size $r$ to be deleted. In the following, the terms $r$-subset and $p$-subset will always refer, respectively, to subsets being deleted and subsets being retained.

The method for selection of best subset is based on $m$-variable reductions, i.e. reductions in the regression sum of squares due to eliminating subsets of size $m$ from the $k$-variable equation. Typically $1 \leq m \leq 4$ and $m=1$ in the original method [5]. These $m$-variable reductions are used to determine the best $r$-subset to be removed, for $r>m$. The reduction in the regression sum of squares due to removing a set of $r$ variables is given by:

$$
\operatorname{Red}_{r}=R S S_{p}-R S S_{k}
$$

The set of $r$ variables for which this reduction is minimum specifies the subset of size $p(p=k-r)$ variables in the regression to be retained for which residual sum of squares is minimum. It is suggested in [5] that $C_{p}$ statistic can also be computed by using this reduction in the following way:

$$
C_{p}=\frac{R e d_{r}}{\hat{\sigma}^{2}}-(2 p-k) .
$$


The steps of the generalized algorithm are as follows [6]: First, the $k$-variable equation is fitted with all variables present and the reductions in the regression sum of squares, called univariate reduction, due to deleting each of the $k$-variables, are calculated. If a single variable, say ith is removed from the regression, its univariate reduction is given by:

$$
\begin{aligned}
\Theta_{i} & =\hat{\sigma}^{2} t_{i}^{2} . \\
t_{i}^{2} & =\frac{b_{i}^{2}}{\hat{\sigma}_{b_{i}}^{2}} .
\end{aligned}
$$

The square of $t$-statistic associated with $i$ th regression coefficient is denoted by $t_{i}^{2}$ and the standard error of the estimated coefficient $b_{i}$ is denoted by $\hat{\sigma}_{b_{i}}$. The standard errors of the estimated coefficients are the square roots of the diagonal elements of the coefficient covariance matrix. After this, the variables are relabeled according to the order of these univariate reductions. Having the variables relabeled, the reductions in the regression sum of squares due to all of the $\left(\begin{array}{c}k \\ m\end{array}\right)$ subsets of size $m$ are evaluated. These are called $m$-variable reductions. Each subset of size $m$ is denoted by the subscripts of the variables in that subset in increasing order. That is, a $m$-subset is described as $\left(i_{1}, i_{2}, \ldots, i_{m}\right)$ where $1 \leq i_{j} \leq k$ and $i_{1} \leq i_{2} \leq \ldots \leq i_{m}$. Next these $m$-variable reductions are ordered in increasing order of magnitude and are used to define stages for inspecting the $r$-subsets. Only those $m$-subsets whose first index is $(r-m+1$ ) or greater are used to define a stage and hence we evaluate only them. The $r$-subsets in a stage defined by an $m$-subset contain $r$ indices consisting of those indices in the defining $m$-subset and $r-m$ indices which are less than the first index in the $m$-subset. There are total of $\left(\begin{array}{c}k-r+m \\ m\end{array}\right)$ stages defined in this way. The stages are numbered according to the magnitude of the $m$-variable subset which define them. Thus Stage 1 will consist of $r$-subsets defined by the $m$-subset with smallest reduction.

In general, at the $q$ th stage we evaluate the reductions due to all subsets defined in the $q$ th stage and ask if the smallest reduction in the regression sum of squares computed for an $r$-subset obtained in all Stages before the $q$ th stage $(1,2, \ldots, q)$ is less than the reduction due to the $m$-subset defining Stage $(q+1)$. If so, we terminate having identified the best subset of size p obtained by the corresponding $r$-subset and if not we proceed to Stage $(q+1)$.

\section{Experiment}

The database for our experiment contains 140 signatures collected from fourteen users. All ten signatures of each user are acquired by using a digitizing tablet Wacom Intuos3 A5 PTZ-630 having a resolution of 5080 lines per inch and an acquisition area of the pad of 152.4 x $210.6 \mathrm{~mm}$ and sampling rate of 200 points per second. Signature raw data is obtained using the .NET Tablet PC SDK 1.7 in a $\mathrm{C} \#$ software program. Having raw data, we evaluate all the features listed in Table 1. 
Table 1. Signature features

\begin{tabular}{|c|c|c|c|c|c|}
\hline$A 1$ & height & $A 2$ & $\begin{array}{l}\text { height } \\
\text { ratio }\end{array}$ & A3 & iumber of points \\
\hline $\begin{array}{l}44 \\
\end{array}$ & $\begin{array}{l}\text { distance between } \\
\text { initial and center } \\
\text { point }\end{array}$ & $A 5$ & $\begin{array}{l}\text { distance } \\
\text { between end and } \\
\text { center point }\end{array}$ & $A 6$ & $\begin{array}{l}\text { angle of the line } \\
\text { between center } \\
\text { and initial points }\end{array}$ \\
\hline$\overline{A r}$ & $\begin{array}{l}\text { angle of the line } \\
\text { between center } \\
\text { and end points }\end{array}$ & $A 8$ & $\begin{array}{l}\text { angle of the line } \\
\text { between initial } \\
\text { and end points }\end{array}$ & $A 9$ & $\begin{array}{l}\text { distance between } \\
\text { leftmost and cen- } \\
\text { ter points }\end{array}$ \\
\hline$\overline{10}$ & $\begin{array}{lr}\text { angle of } & \text { the line } \\
\text { between } & \text { center } \\
\text { and } & \text { leftmost } \\
\text { points } & \end{array}$ & $A 11$ & \begin{tabular}{|lr} 
angle of the line \\
between \\
and renter \\
points
\end{tabular} & $A 12$ & $\begin{array}{l}\text { distance between } \\
\text { leftmost and ini- } \\
\text { tial points }\end{array}$ \\
\hline 13 & $\begin{array}{l}\text { distance between } \\
\text { rightmost and } \\
\text { end points }\end{array}$ & $A 14$ & $\begin{array}{l}\text { angle of the line } \\
\text { angle of the line } \\
\text { between leftmost } \\
\text { and initial points }\end{array}$ & $A 15$ & $\begin{array}{l}\text { angle of the line } \\
\text { between end and } \\
\text { rightmost points }\end{array}$ \\
\hline
\end{tabular}

The number of features is $k=15$ and the number of signatures is $n=20$ (ten genuine and ten random forgeries). For each user we construct a txt file. Each line of that file contains signature features values separated by a semicolon followed by 1 (if genuine, i.e. first class) or -1 (if forgery, i.e. second class). The random forgeries for a given users are just randomly selected signatures of the rest users. Our software program processes each of these fourteen files by performing the method described in Section 2 and finds the best subset of size $p(p=k-r)$ for variable values of $r(3<r<13)$. Among these subsets we select the subset that have $C_{p}$ value closest to $p$. These subsets for each user are listed in Table 2 .

Table 2. Best p-subsets for each user

\begin{tabular}{|c|c|c|}
\hline User No & Size of p-subset & Best $\boldsymbol{p}$-subset \\
\hline 1 & 6 & $\mathrm{~A} 2 ; \mathrm{A} 5 ; \mathrm{A} 7 ; \mathrm{A} 12 ; \mathrm{A} 13 ; \mathrm{A} 15$ \\
\hline 2 & 9 & $\mathrm{~A} 3 ; \mathrm{A} 5 ; \mathrm{A} 6 ; \mathrm{A} 7 ; \mathrm{A} 8 ; \mathrm{A} 9 ; \mathrm{A} 10 ; \mathrm{A} 11 ; \mathrm{A} 12$ \\
\hline 3 & 11 & $\mathrm{~A} 2 ; \mathrm{A} 4 ; \mathrm{A} 5 ; \mathrm{A} 6 ; \mathrm{A} 7 ; \mathrm{A} 8 ; \mathrm{A} 9 ; \mathrm{A} 10 ; \mathrm{A} 13 ; \mathrm{A} 14 ; \mathrm{A} 15$ \\
\hline 4 & 11 & $\mathrm{~A} 1 ; \mathrm{A} 3 ; \mathrm{A} 4 ; \mathrm{A} 6 ; \mathrm{A} 7 ; \mathrm{A} 8 ; \mathrm{A} 9 ; \mathrm{A} 11 ; \mathrm{A} 12 ; \mathrm{A} 13 ; \mathrm{A} 15$ \\
\hline 5 & 5 & $\mathrm{~A} 1 ; \mathrm{A} 2 ; \mathrm{A} 5 ; \mathrm{A} 9 ; \mathrm{A} 15$ \\
\hline 6 & 6 & $\mathrm{~A} 1 ; \mathrm{A} 2 ; \mathrm{A} 5 ; \mathrm{A} 10 ; \mathrm{A} 12 ; \mathrm{A} 14$ \\
\hline 7 & 7 & $\mathrm{~A} 1 ; \mathrm{A} 2 ; \mathrm{A} 3 ; \mathrm{A} 4 ; \mathrm{A} 5 ; \mathrm{A} 8 ; \mathrm{A} 9 ; \mathrm{A} 10 ; \mathrm{A} 15$ \\
\hline 8 & 9 & $\mathrm{~A} 2 ; \mathrm{A} 5 ; \mathrm{A} 6 ; \mathrm{A} 7 ; \mathrm{A} 8 ; \mathrm{A} 9 ; \mathrm{A} 11 ; \mathrm{A} 12 ; \mathrm{A} 13 ; \mathrm{A} 14 ; \mathrm{A} 15$ \\
\hline 9 & 11 & $\mathrm{~A} 4 ; \mathrm{A} 6 ; \mathrm{A} 9 ; \mathrm{A} 10 ; \mathrm{A} 11 ; \mathrm{A} 12 ; \mathrm{A} 13 ; \mathrm{A} 14$ \\
\hline 10 & 8 & $\mathrm{~A} 1 ; \mathrm{A} 3 ; \mathrm{A} 4 ; \mathrm{A} 5 ; \mathrm{A} 6 ; \mathrm{A} 7 ; \mathrm{A} 9 ; \mathrm{A} 11 ; \mathrm{A} 13$ \\
\hline 11 & 9 & $\mathrm{~A} 1 ; \mathrm{A} 2 ; \mathrm{A} 3 ; \mathrm{A} 4 ; \mathrm{A} 5 ; \mathrm{A} 6 ; \mathrm{A} 7 ; \mathrm{A} 9 ; \mathrm{A} 10 ; \mathrm{A} 11 ; \mathrm{A} 15$ \\
\hline 12 & 11 & $\mathrm{~A} 1 ; \mathrm{A} 2 ; \mathrm{A} 3 ; \mathrm{A} 5 ; \mathrm{A} 6 ; \mathrm{A} 8 ; \mathrm{A} 10 ; \mathrm{A} 11 ; \mathrm{A} 12 ; \mathrm{A} 14$ \\
\hline 13 & 10 & $\mathrm{~A} 1 ; \mathrm{A} 2 ; \mathrm{A} 3 ; \mathrm{A} 4 ; \mathrm{A} 5 ; \mathrm{A} 7 ; \mathrm{A} 8 ; \mathrm{A} 9 ; \mathrm{A} 10 ; \mathrm{A} 11$ \\
\hline 14 & 10 &
\end{tabular}


These results demonstrate that the number of features is significantly reduced for some users but there are not common features among best feature subsets for all users which will justify the removal of the rest features from the initial feature set. That is because all the features have approximately equal number of occurrences in best subsets. The results demonstrated that we cannot restrict to common feature set valid for all users but instead of that we have to consider each user best feature set separately in signature verification.

\section{Conclusion}

In this paper we present a method for feature set selection based on Mallows $\mathrm{Cp}$ criterion for regression and conduct an experiment in order to test it with a database of 140 signatures from fourteen users. The obtained results demonstrate that this approach can be used for feature set reduction for a particular user before his/her signature verification. Probably the small number of features does not allow the presence of common features for all users. Therefore, the focus of our future work will be the application of the method for selection of regression variables for larger feature set. Also, we will experiment with skilled signature forgeries and investigate the problem further.

\section{Acknowledgements}

This work is supported by the European Social Fund and Bulgarian Ministry of Education, Youth and Science under Operative Program Human Resources Development, Grant BG051PO001-3.3.04/40.

\section{References}

1. Nalwa, V.S., Ekeland, I.: Automatic on-line signature verification. Proceedings of the IEEE 85, 213-239 (1997)

2. Chatterjee, S., Hadi, A.: Regression Analysis by Example, 4th edn. New York (2006)

3. Leclerc, F., Plamondon, R.: Automatic signature verification: the state of the art 1989-1993. International Journal of Pattern Recognition and Artificial Intelligence 8, 643-660 (1994)

4. Richiardi, J., Ketabdar, H., Drygajlo, A.: Local and Global Feature Selection for On-line Signature Verification. In: Eighth International Conference on Document Analysis and Recognition (ICDAR 2005), pp. 625-629 (2005)

5. Hocking, R.R., Leslie, R.n.: Selection of the Best Subset in Regression Analysis. Technometrics 9, 531-540 (1967)

6. LaMotte, L.R., Hocking, R.R.: Computational Efficiency in the Selection of Regression Variables. Technometrics 12, 83-93 (1970)

7. Hocking, R.R.: The Analysis and Selection of Variables in Linear Regression. Biometrics 32, 1-50 (1976)

8. Mallows, C.L.: Some Comments on Cp. Technometrics 15, 661-675 (1973) 